\title{
Gene Therapy For Adenosine Deaminase Deficiency: Successes and Limitations
}

\author{
Michael Ga-Hong Woo, B.Sc. ${ }^{* \dagger}$
}

\section{INTRODUCTION}

Severe combined immunodeficiency disease (SCID), often dubbed the "baby in a bubble" syndrome, represents the most severe type of primary immunodeficiencies (1). It is a heterogeneous group of congenital disorders caused by a number of different defects of the lymphoid lineages (2) and the estimated incidence is 1 in 100,000 live births (3). Several natural mutants have been characterized in humans, all of which involve complete block of T-cell development, and will directly or indirectly impair B-cell immunity $(1,4)$. This leads to devastating clinical symptoms as a result of predisposition to infections from many opportunistic pathogens (4). In many cases, severe infections starting at 1 to 3 months of age will lead to death if untreated $(5,6,7)$. This disease received worldwide recognition in the 1970 s when the story of David Vetter, who lived all of his 12 years of life inside a sealed plastic bubble designed to protect him from infections was brought to light. Subsequently, his life story and the unique strategies used to treat David, received widespread media attention, being the subject of movies and television shows .

The adenosine deaminase (ADA) deficient variant of

* To whom correspondence should be addressed: Michael Ga-Hong Woo, St. Boniface Hospital Research Centre, DNND Rm. 4046 351 Tache Ave.Winnipeg, Manitoba, R2H 2A6, Canada. E-mail: mwoo@sbrc.ca

$\dagger$ Division of Neurovirology and Neurodegenerative Disorders, St. Boniface Hospital Research Centre and Department of Pharmacology and Therapeutics, University of Manitoba, Winnipeg, Manitoba.
SCID is an autosomal recessive disorder and accounts for approximately $20 \%$ of all SCIDs (8). This inherited deficiency results in decreased enzymatic activity or the lack of production of adenosine deaminase a housekeeping enzyme of the purine salvage pathway (9, 10). ADA was the first gene associated with a SCID condition to be identified (11), and was the focus of the first gene therapy trial in 1990 (12). ADA-SCID is a lethal disorder that is now treated with either allogeneic bone marrow transplantation or enzyme replacement therapy $(2,13)$. Gene therapy of this disease is in clinical trials and has produced the most promising clinical experience thus far of all the genetic diseases. ADA-SCID patients have been transplanted with transduced autologous $\mathrm{T}$ lymphocytes and hematopoietic stem cells (HSC) $(4,7)$. The progress made in the attempt to treat this disease reflects both the successes of gene therapy and the limitations of it that have to be overcome before it can be a reliable and realistic treatment for ADA deficiency and other genetic diseases. The past, present and future therapies of ADASCID will be examined to demonstrate this progress.

\section{WHAT IS ADENOSINE DEAMINASE?}

Adenosine deaminase (ADA) is an important deaminating enzyme of the purine salvage pathway that converts adenosine and 2'-deoxyadenosine to inosine and 2'-deoxyinosine respectively $(2,11,12,14)$. ADA allows for the conversion of adenosine into other purines to be recycled and removed by formation of uric acid, which is the end product of purine metabolism in humans (14). ADA is especially critical for cells such as 
lymphocytes and erythrocytes that lack or have very low activity of the de novo purine synthetic pathway (2).

In humans, there are two isoforms of the ADA enzyme, adenosine deaminase 1 (ADA1) and adenosine deaminase 2 (ADA2). Intracellular ADA activity is mainly mediated by ADA1, while ADA2 is the predominant isoform in human plasma and serum (14). The cellular source of the latter has been linked to the monocyte-macrophage cell system, although it is widely accepted that ADA2 activity represents T-cell function, and measurements of ADA2 activity has been used to evaluate the disease severity of patients with acquired immunodeficiency syndrome (14).

The importance of maintaining normal levels of ADA activity can be seen in patients with a genetic deficiency of this enzyme. Lack of ADA activity, which is important in T-cell development, is associated with a form of severe combined immunodeficiency disease referred to as ADA-SCID $(11,14)$. In terms of biochemistry, the lymphospecific toxicity associated with this disease is thought to be the result of the accumulation of 2'-deoxyadenosine (a substrate of ADA) and its conversion to the phosphorylated form (deoxyadenosine triphosphate, dATP), which is an inhibitor of ribonucleotide reductase, a key enzyme in DNA synthesis and DNA repair in dividing T cells (15). This leads to cell death in non-dividing T cells $(1,14)$. A second mechanism contributing to the pathology of ADA-SCID involves the methylation reactions of Sadenosylmethionine to S-adenosylhomocysteine (AdoHcy) (14). AdoHcy is hydrolyzed to adenosine and homocysteine by AdoHcy hydrolase. In patients with ADA-SCID, accumulating 2'-deoxyadenosine inhibits AdoHcy hydrolase resulting in the accumulation of AdoHcy. AdoHcy then functions as a competitive inhibitor of many transmethylation reactions critical to cellular functions (14).

\section{CLINICAL AND PATHOLOGIC FEATURES OF ADA-SCID}

Classically, SCID is defined as a fatal infantile syndrome with symptoms resulting from the absence of cellular and humoral immunity. Most infants suffering from ADA-SCID have shown the same clinical and immunological manifestations as patients with nonADA-SCID $(16,17)$. Although these infants suffer from lymphopenia and absence of non-maternally derived immunoglobulin, symptoms may not appear until several weeks to several months of life (2). The fullblown syndrome includes overwhelming fungal, viral and bacterial infections and failure to thrive (10).

While the underlying immunodeficiency in ADASCID appears early in life, there is a progressive worsening of the condition as toxic metabolites accumulate (due to the absence of ADA) and continue to interfere with normal T-cell and B-cell function. Although $15 \%$ of ADA-SCID patients show a slightly later onset and a slower progression of symptoms, it is still fatal. The variability of disease progression may be partly a result of environmental factors and undoubtedly of genetic origin. If left untreated, ADA-SCID is fatal by 1-2 years of age; however, it is more common for death to occur during the first few months of life (2).

In addition to infantile-onset ADA-SCID, cases of later onset immunodeficiency have also been reported (2). In one of the earliest cases of ADA deficiency, clinical symptoms did not appear until two years of age and the only detectable abnormality suggestive of an immunodeficient phenotype, prior to disease onset, was a phasic appearance of lymphopenia and eosinophilia (18). Since then, other cases have been reported and reviews of patient medical history and laboratory findings reveal common features. At the time of diagnosis, all had diminished $\mathrm{T}$ cell counts with low mitogen responses; however, several showed normal total Ig and antibody responses to some antigens (19, $20,21)$. They had substantially high IgE and/or eosinophilia, a history of recurring sinopulmonary bacterial infections including pneumococcal pneumonia and septicemia, and inability to produce antibody to some antigens such as pneumococcus $(19,20,21)$. An interesting feature seen in two cases was the diagnosis of "autoimmune" hypothyroidism $(20,21)$, which could directly reflect toxicity to the thyroid or autoimmune disease due to abnormal regulation of the immune response. These later onset ADA-SCID patients would show more residual ADA activity than those with the infantile-onset disease (2).

\section{CURRENT TREATMENTS FOR ADA-SCID Bone Marrow Transplantation}

The current curative treatment of choice for all SCID patients including ADA-SCID is bone marrow transplantation (BMT) from an HLA-identical sibling $(11,12,13)$. HLA, an abbreviation of human leukocyte antigen, is the major histocompatibility antigen occurring on human nucleated cells, including lymphocytes. This form of treatment results in a longterm cure rate of $95-100 \%$ (22), however, less than one third of patients have access to an HLA-identical donor $(11,12)$. In the absence of an HLA-identical sibling donor, T-cell depleted parental bone marrow (haploidentical donor) is preferred over an unrelated donor. This alternative has provided less encouraging results and reports have shown that treatment success rates of BMT for ADA-SCID patients lacking an HLAgenotypically identical donor have not improved over the last 20 years (12). Significant side effects can result due to the need for conditioning cytoreduction and 
immunosuppression with systemic chemotherapy and total body irradiation, which increases the risk of both short and long term complications. Complications include life-threatening infections, acute cardiomyopathy, progressive pulmonary fibrosis, irreversible sterility and secondary malignancies (10). For all these reasons, BMT is not a useful treatment for all SCID patients, especially those who are too sick to tolerate cytoreductive therapy or where the risks associated with this treatment is felt to be too high (10).

\section{Polyethylene Glycol-modified Bovine ADA (PEG- ADA)}

For patients with ADA-SCID who are lacking an HLA-identical bone marrow donor, an alternative treatment would be enzyme replacement therapy using polyethylene glycol-modified bovine ADA (PEG-ADA) (23). Because the toxic substrates of ADA (adenosine and deoxyadenosine) diffuses freely throughout the body, injecting this enzyme into ADA-deficient patients can replace the function of the missing enzyme (24). Infusions of purified bovine ADA linked to polyethylene glycol have been successful in decreasing the number of infections by increasing lymphocyte count and by restoring partial T-cell function. However, the formation of inactivating antibodies against the bovine ADA has been observed, and full immune reconstitution is less regularly achieved with this therapy $(4,8,25)$. This treatment is also very expensive, costing an estimated US $\$ 250,000 / \mathrm{yr}$./patient $(11,26)$.

In a study by Bordignon et al. (1993), two children suffering form ADA-SCID were treated with PEG enzyme replacement therapy and showed very promising initial results (10). Weekly doses of $20 \mathrm{U} / \mathrm{kg}$ body weight resulted in a therapeutically constant plasma level abolishing all the tested biochemical abnormalities associated with ADA deficiency. Improvements included the absence of infections and restored weight and height gain, and after three months of therapy, the patients did not require isolation or hospitalization. During these three months, their absolute lymphocyte counts normalized, as well as percentages of $\mathrm{CD} 3+, \mathrm{CD} 4+, \mathrm{CD} 8+\mathrm{T}$ cell populations, and lymphocyte proliferative responses to PHA and IL2 , indicating an improved immune system. One patient, upon receiving vaccination from tetanus toxoid and FSME virus one year after initial PEG-ADA treatment, developed specific antibodies and produced specific Tcells to both antigens. Unfortunately, these improvements did not persist after discontinuation of i.v. immunoglobulin prophylaxis, with the appearance of decreased total lymphocyte counts, reduction of TCR repertoire and antigen specific responses. In addition, results indicated that intracellular production of ADA activity would be more efficient in promoting lymphocyte survival and immune functions rather than extracellular detoxification as in the case with PEGADA replacement therapy.

\section{RATIONALE FOR THE GENE THERAPY OF SEVERE COMBINED IMMUNODEFICIENCIES (SCID)}

Over the past 3 decades, advances in molecular biology have demonstrated the usefulness of gene therapy as a new tool to correct patient cell function and to alleviate disease. The first successful gene transfer with a retroviral vector to murine hematopoietic cells was reported in 1983 (27), and since then, numerous studies using murine hematopoietic stem cells have been done to assess the potential use for human stem cell gene therapy (12). Encouraging murine in vivo studies in which recombinant murine retroviruses infect murine hematopoietic stem cells, have demonstrated high efficiency (28). More importantly, these cells had the ability to maintain long-term expression of the transduced gene. These successes led to the belief that human stem cell gene therapy could soon be a reliable treatment for various congenital or acquired human diseases.

There are a number of reasons that have made this disease a primary focus for gene therapy. First, ADA deficiency is the most extensively studied congenital immunodeficiency disease (12). The genomic and cDNA sequences encoding ADA were identified early on $(29,30)$, and the structure and function of this enzyme is well understood (12). Second, it is a disease that if left untreated, results in debilitating and lethal effects. Because an alternative effective therapy is currently not available for every patient, the potential risk of gene therapy experimentation becomes more acceptable. Third, it is known that patients receiving bone marrow transplantation can be cured of this disease $(9,31)$, and so the target tissue for the introduction of the ADA gene is the easily accessible hemopoietic system. Fourth, based on various studies, it is expected that the genetically corrected cells should persist and should have a selective growth advantage over the non-transduced cells in vivo after transplantation $(32,33)$. Finally, the regulation of the ADA gene would not have to be precisely regulated nor would the expression have to be cell-type specific for beneficial effects to occur. Patients exhibiting $10 \%$ of normal ADA levels do not show any apparent immune impairments (34) while patients with ADA activity greater than 50-fold above normal suffer only from a moderate hemolytic anemia (35). If the ADA gene can be introduced in pluripotent hemopoietic stem cells 
(PHSC), reimplantation and normal growth of these transduced cells could result in a life-long production of corrected immune cells.

\section{GENE THERAPY FOR ADENOSINE DEAMINASE DEFICIENCY}

Gene therapy involves the introduction of exogenous genetic material to correct or modify the function of a cell. It is an emerging medical procedure where genetic diseases could be corrected by transfer of a normal version of a relevant gene into a patient's somatic cells. Gene transfer into a patient's hematopoietic stem cells followed by their autologous transplantation could provide the same benefits as allogenic transplantation without the immunological complications such as graft rejection, graft versus host disease, and posttransplantation immunosuppressive therapy. Although gene therapy to treat blood diseases seems logical, there are still more problems than successes, mainly from inadequate tools used for gene transfer and gene expression. However, despite bouts of successes and failures, techniques for gene transfer, gene expression and hematopoietic stem cell manipulation have steadily improved.

To date, a number of clinical gene transfer trials using human hematopoietic stem cells (HSCs) have been performed worldwide to test the potential of human stem cell gene therapy to treat $\operatorname{ADA}-\operatorname{SCID}(7,36,37)$. However, the results from these trials are somewhat disappointing, revealing that murine studies cannot always apply to humans. The transduction frequencies in human HSCs were low and clinical benefits were not apparent in all cases. In spite of these failures, many lessons were learned that could be applied to gene therapy of other genetic diseases.

The lessons learnt from preclinical studies and subsequent early clinical trials for ADA-SCID demonstrate the advances in gene therapy made so far. Preclinical studies in the 1980's showed that murine hematopoietic stem cells (HSCs) could be transduced in vitro using the Moloney murine leukemia virus (MoMLV)-based retroviral vector, containing the human copy of the ADA gene $(9,38)$. These cells were subsequently transplanted into irradiated mice resulting in circulating lymphohematopoietic cells that carried and expressed the human ADA gene $(9,38)$. These preclinical results suggested that a similar strategy could be applied in humans to successfully treat patients with ADA deficiency. As a result, researchers proposed to harvest autologous bone marrow from ADA-SCID patients, to transduce these cells in vitro with a retroviral vector carrying the normal human ADA gene, and to infuse these cells into the respective patients without using pretransplant myeloablative chemotherapy.
Although it was a good idea, researchers at the time lacked in vitro and in vivo systems to evaluate human HSC gene transduction, so preclinical research could not definitively conclude that human HSC could be transduced (9). Instead, many researchers turned their attention to the transduction of peripheral blood $\mathrm{T}$ lymphocytes of ADA-deficient patients who were receiving PEG-ADA enzyme replacement therapy. This different approach was a result of preclinical studies showing that in vitro transduction of peripheral blood $\mathrm{T}$ lymphocytes from ADA-SCID patients, using a retroviral vector, was possible (33). The genetically modified lymphocytes were transplanted intraperitoneally into immunodeficient mice, and were examined one month later. Results indicated that $\mathrm{T}$ lymphocytes, which had been transduced with the normal human ADA gene, persisted, while T lymphocytes transduced with a control vector did not (33). This indicated that transduction of the normal ADA gene into ADAdeficient $\mathrm{T}$ lymphocytes was a feasible approach to treating ADA-SCID $(10,24,33)$.

These preclinical experiments were very encouraging and led investigators at the National Institutes of Health (NIH) to use a similar strategy to treat humans. In 1990, the Blaese group at the NIH performed the first clinical gene therapy trial on 2 young female patients with ADA-SCID $(4,12)$. They targeted peripheral blood $\mathrm{T}$ lymphocytes from the patients with ADA-SCID on PEG-ADA therapy with a MoMLV-based retroviral vector containing the normal human ADA gene (LASN) (4). The patient's lymphocytes were harvested, genetically modified, expanded more than 50-fold, and given back by infusion (4). Over 2 years, the patients received a total of 11-12 infusions of autologous genetically corrected lymphocytes and as a result, immune functions of both patients were better than when only on PEG-ADA treatment alone $(4,12)$. Unfortunately, the transduction frequency of the infused $\mathrm{T}$ lymphocytes differed in the two patients $(30 \%$ and $<1 \%)$ and they also continued to receive PEG-ADA therapy throughout the procedure (4). These experiments were able to show that transduction of human peripheral blood $\mathrm{T}$ lymphocytes are possible and that their progeny could persist in vivo for many years. These experiments also raised many questions such as; what was the antigenic repertoire of the transduced cells? More importantly, could the transduced T-cells persist without the exogenous source of ADA that was coming from the PEG-ADA therapy?

As a result of these questions, many groups had the ambitious goal to permanently correct ADA deficiency by genetically correcting autologous haematopoietic stem cells (HSC). If successful, they would avoid the possible problem of defects in the antigenic repertoire 
of the mature $\mathrm{T}$ cell used in previous transplantations, and only one infusion of genetically corrected stem cells would be needed to restore the patient's immune functions (23). Three clinical trials have been conducted examining the use of transduced autologous HSC to treat ADA deficiency. One trial used only bone marrow HSC, another used bone marrow HSC in addition to peripheral blood $\mathrm{T}$ lymphocytes, and the last used umbilical cord blood HSC $(9,24)$.

The group conducting the clinical trial using CD34+ cells from bone marrow alone, reported that the transduction efficiency was disappointingly low and that transduced peripheral leukocytes could not be detected in the long-term $(9,12,37)$.

On the other hand, in 1992, Bordignon et al., using transduced CD34+ bone marrow cells and PEG-ADA dependent $\mathrm{T}$ lymphocytes, showed rapid improvements in patient immune functions after gene therapy (7). Bordignon's group used two different retroviral vectors to transduce a normal ADA cDNA separately into peripheral T-lymphocytes or bone marrow cells to determine which cell population was the major source of the circulating peripheral blood $\mathrm{T}$ lymphocytes. Their results show that immediately after transplantation the circulating transduced $\mathrm{T}$ cells originated from the infused peripheral $\mathrm{T}$ lymphocytes, but as time passed they were replaced with transduced $T$ cells derived from the transduced bone marrow cells $(7,9)$. This suggests that bone marrow HSC can be transduced and that genetically modified HSC can give rise to functional mature cells detectible in the peripheral circulation. Unfortunately, the patients in this trial continued to receive $\mathrm{PEG}-\mathrm{ADA}$ enzyme replacement therapy, so it remains unclear if transduction of $\mathrm{T}$ lymphocytes of HSC origin can provide clinical benefits to ADA-SCID (9).

The third study used CD34+ cells from umbilical cord blood $(9,12,36)$. Three neonates were diagnosed in utero with ADA deficiency and cells were transduced with the same LASN vector used in Bordignon's study (9). Transduction of progenitor cells was efficient and longitudinal evaluation of the patients for transduced leukocytes occurred. A year after transplantation, the group reported low but sustained levels of transduced cells in mononuclear cells and granulocytes and increased ADA activity in HSC (36). These patients started PEG-ADA therapy during their first week of life and showed characteristics of a normal functioning immune system. At age 2, PEG-ADA treatment doses were decreased, resulting in a significant decrease in circulating $\mathrm{T}$ lymphocytes and a 100-fold increase in the frequency of $\mathrm{T}$ lymphocyte transduction without change in myeloid cells or B cells (36). As the dose of PEGADA decreased, a selective advantage in survival of the transduced $T$ cells originating from the transduced HSC occurred, but was nonexistent in B cells or other hematopoietic cells $(24,36)$. At age 5, PEG-ADA therapy was discontinued in one patient, and over a twomonth period, plasma ADA levels of this patient became undetectable, levels of ADA substrates increased, and there was substantial decrease in percentage and absolute levels of natural killer (NK) cells and B lymphocytes although no changes in levels of T lymphocytes occurred (36). Again, results showed a selective advantage in survival of the transduced $\mathrm{T}$ cell progeny of the transduced HSC. In addition, analysis of $\mathrm{T}$ cell function revealed a loss of antigenspecific blastogenesis to tetanus toxoid and candida (9). Interestingly, results also showed that expression of the MoMLV-based retroviral vectors were low in resting human $\mathrm{T}$ cells but were relatively high in dividing $\mathrm{T}$ lymphocytes, indicating that LASN vector is expressed during thymopoiesis and not in resting peripheral blood T lymphocytes (9). Results suggest that cessation of PEG-ADA therapy resulted in the loss of both transduced and nontransduced antigen-specific peripheral blood $\mathrm{T}$ lymphocytes. Since the patient started to show clinical symptoms of immune deficiency such as weight loss, oral thrush and upper respiratory infection, the patient resumed PEG-ADA therapy, resulting in restoration of good health $(9,36)$. The results obtained from this trial suggest that cord blood provides a stem cell population more suitable for efficient retroviral-mediated gene transfer than does bone marrow, however, significant advances are still needed in this transfer technique before human HSCs can be used to restore effective immunity and to achieve clinical benefits (11).

\section{CONCLUSION}

The current clinical results of gene therapy for ADASCID are encouraging but also reveal current limitations of gene therapy for immunodeficiency disorders. Results have shown that transduction and transplantation of HSC from both bone marrow and cord blood is possible, and that transduction frequency in $\mathrm{T}$ lymphocytes can reach as high as $10-30 \%$ if a selection advantage existed in vivo. Unfortunately, when an exogenous source of ADA was removed, results showed a lack of gene expression in nondividing $\mathrm{T}$ lymphocytes, indicating the loss of both transduced and nontransduced antigen-specific peripheral blood $\mathrm{T}$ lymphocytes. Successful treatment of SCID or diseases involving lymphoid differentiation will ultimately require expression of vectors in mature, nondividing lymphoid cells. For this reason, many investigators are focusing on improving gene transfer technologies. Recent improvements have resulted in the development of better vectors, packaging cell lines and culture conditions for human HSC transduction. For example, use of certain cytokines and recombinant fibronectin has improved transduction efficiency of HSCs in 
baboon and rhesus monkeys $(39,40)$. These cytokines can induce cycling of immature CD34+ cells making them more receptive to transgene integration. Also, new packaging cell lines have been designed which enhances binding of retrovirus to hematopoietic cells by pseudotyping with the gibbon ape leukemia virus envelope resulting in increased rate of $\mathrm{CD} 34+$ cell transduction (41). To reduce risk of in vivo transgene silencing, deletion of silencing sequences from viral LTR has been examined. Design of lentiviral vectors have also provided encouraging results, being able to infect non-cycling cells. Although attempts to cure ADA-SCID with gene therapy have yet to prove successful, study of this disease has resulted, by far, in the most promising clinical experience by identifying current limitations and providing enough successes to encourage the pursuit of the solutions.

\section{REFERENCES}

1. The Boston Women's Health Book Collective. Our Bodies, Ourselves. New York: Simon and Schuster, 1998.

2. An Article in Calgary Herald, May 19, 1998 as quoted by Gunhild Hoogensen "The Politics of Birth: Midwifery in Alberta" 2000. www.birthpartnershipmidwives.com

3. Buske, Lynda. " A Crisis Aborning in Newborn and Maternity Care?" Canadian Medical Association Journal, March 6, 2001; 164 (5).

4. Chan, Benjamin TB "From Percieved Surplus to Percieved Shortage: What Happenned to Canada's Physician Workforce in the 1990's?" Canadian Institute for Health Information, June 2002.

5. Blais, Regis "Commentary/Commentaire: Are Home Births Safe?" Canadian Medical Association Journal, February 5, 2002; 166 (3).

6. Legislation Concerning Nursing/Midwifery Services and Education (EURO Reports and Studies, 1981) as quoted in Blais, Maheux, Lamber, Loiselle, Gauthier, Framarin "Midwifery defined by physicians, nurses and midwives: The birth of consensus?" Canadian Medical Association Journal 1994; 150 (5): 691-697.

7. Bailey, Lehr, Nicholas, Picco "Midwifery: Promotion and integration into Canada's healthcare system" Leadership in Health Services July/August 1993: 11-13.7.

8. Bourgeault, Ivy Lynn and Mary Fynes "Integrating Lay and Nurse Midwifery into the U.S. and Canadian Health Care Systems" Social Sciences \& Medicine"1997; 44 (7): 1051-1063.

9. McKendry, Rachael and Tom Langford "Legalized, regulated, but unfounded: midwifery's laborious professionalization in Alberta, Canada, 1975-99" Social Science \& Medicine, 2001;53: 531-542.

10. Canadian Midwifery Regulators Consortium, Submission to the Commission on the Future of Health Care in Canada "Regulated Midwifery and The Future of Health Care in Canada" 2001.

11. Reinharz D, Blais R, Fraser W, Constandriopolous AP "Costeffectiveness of midwifery services vs. medical services in Quebec, L'Equipe d'Evaluation des Projets-Pilotes SagesFemmes" Canadian Journal of Public Health 2000; 91(1): 112115.

12. Janssen, P, Lee, SK, Ryan, E, Etches, D, Farquarson, D, Peacock,D and Klein, M. "Outcomes of planned home births versus planned hospital births after regulation of midwifery in British Columbia" Canadian Medical Association Journal 2002;166 (3):315-323. 12i. Low risk births can be identified at any stage of gestation, however in the Janssen et al study, 3.6\% of homebirths had to call for emergency transports in spite of the application of the following exclusion criteria including multiple birth, heart disease, hypertensive chronic renal disease, pregnancy induced hypertension with protein uria, insulin dependant diabetes, antepartum hemmorhage after 20 weeks gestation, active general herpes, breech or abnormal presentation, gestational age of less than 30 weeks or greater than 41 weeks at the onset of labour, more than one previous cesarian section, and mother transferred to hospital from another health care facility. (Janssen et al.)

13. www.aom.on.ca

14. Harvey,S, Rach, D, Stainton, MC, Jarrell, J, Brant, R. "Evaluation of Satisfaction of midwifery care" Midwifery 2002; 18: 260-267.

15. Ontario Association of Midwives (AOM) as quoted by Scott Piatkowski "Midwifery Remains a Safe Alternative", June 10, 2002. www.straightgoods.ca

16. Blais, Maheux, Lamber, Loiselle, Gauthier, Framarin "Midwifery defined by physicians, nurses and midwives: The birth of consensus?" Canadian Medical Association Journal 1994; 150 (5): 691-697.

17. www.aom.on.ca/midwifery/NowandThen $/ \mathrm{html}$

18. www.asac.ab.ca

19. Bennett, Carolyn. Kill or Cure? Toronto: HarperCollins Publishers Ltd.,2000.

2. Hirschhorn R. Adenosine deaminase deficiency. Immunodeficiency Reviews 2(3):175-98; 1990

3. Kalman L, Lindegren ML, Kobrynski L, et al. IL2RG and Severe Combined Immunodeficiency (SCID), a Primary Immunodeficiency Disease (PID): fact sheet. Human Genome Epidemiology Network, Centers for Disease Control; July 2002 http://www.cdc.gov/genomics/hugenet/factsheets/FS_IL2RG_S cidPid.htm

4. Blaese RM, Culver KW, Miller AD, et al. T lymphocyte-directed gene therapy for ADA-SCID: initial trial results after 4 years. Science 270(5235):475-80; 1995

5. Rosen FS. Successful Gene Therapy for Severe Combined Immunodeficiency. The New England journal of medicine 346(16):1241-43; 2002

6. Hershfield MS. Adenosine deaminase deficiency: clinical expression, molecular basis, and therapy. Seminars in hematology 35(4):291-8; 1998

7. Bordignon C, Notarangelo LD, Nobili N, et al. Gene therapy in peripheral blood lymphocytes and bone marrow for ADAimmunodeficient patients. Science 270(5235):470-5; 1995

8. Aiuti A. Advances in gene therapy for ADA-deficient SCID. Current Opinion in Molecular Therapeutics 4(5):515-22; 2002

9. Parkman R, Weinberg K, Crooks G, Nolta J, Kapoor N, Kohn D. Gene therapy for adenosine deaminase deficiency. Annual Review of Medicine 51:33-47; 2000

10. Bordignon C, Mavilio F, Ferrari G, et al. Transfer of the ADA gene into bone marrow cells and peripheral blood lymphocytes for the treatment of patients affected by ADA-deficient SCID. Human Gene Therapy 4(4):513-20; 1993

11. Onodera M, Ariga T, Kawamura N, et al. Successful peripheral T-lymphocyte-directed gene trasfer for a patient with severe combined immune deficiency caused by adenosine deaminase deficiency. Blood 91(1):30-6; 1998

12. Onodera M, Melson DM, Sakiyama Y, Candotti F, Blaese RM. Gene therapy for severe combined immunodeficiency caused by adenosine deaminase deficiency: improved retroviral vectors for clinical trials. Acta Haematol. 101(2):89-96; 1999

13. Chen SH, Ochs HD, Scott CR, et al. Adenosine deaminase deficiency: disappearance of adenine deoxynucleotides from a patient's erythrocytes after successful marrow transplantation. 
The Journal of clinical investigation 62(6):1386-9; 1978

14. Cristalli G, Costanzi S, Lambertucci C, et al. Adenosine deaminase: Functional implications and different classes of inhibitors. Medicinal Research Reviews 21(2):105-28; 2001

15. Chabes AL, Pfleger CM, Kirschner MW, et al. Mouse ribonucleotide reductase $\mathrm{R} 2$ protein: A new target for anaphasepromoting complex-Cdh1-mediated proteolysis. Proceedings of the National Academy of Sciences of the United States of America 100(7):3925-9; 2003

16. Hirschhorn R. Inherited enzyme deficiencies and immunodeficiency: adenosine deaminase (ADA) and purine nucleoside phosphorylase (PNP) deficiencies. Clinical immunology and immunopathology 40(1):157-65; 1986

17. Meuwissen HJ, Pollara B, Pickering RG, et al. Combined immunodeficiency disease associated with adenosine deaminase deficiency. The Journal of pediatrics 86:169-81; 1975

18. Giblett ER, Anderson JE, Cohen F, et al. Adenosine-deaminase deficiency in two patients with severely impaired cellular immunity. Lancet 2(7786):1067-9; 1972

19. Levy Y, Hershfield MS, Fernandez-Mejia C, et al. Adenosine deaminase deficiency with late onset of recurrent infections: response to treatment with polyethylene glycol-modified adenosine deaminase. The Journal of pediatrics 113(2):312-7; 1988

20. Cowan MJ, Martin DW Jr, Warra DW, et al. Intravenous deoxycytidine therapy in a patient with adenosine deaminase deficiency. Advances in experimental medicine and biology 165 Pt A:39-45; 1984

21. Geffner ME, Stiehm ER, Stephure D, et al. Probable autoimmune thyroid disease and combined immunodeficiency disease. American journal of diseases of children (1960) 140(11):1194-6; 1986

22. Hirschhorn R. Adenosine deaminase deficiency. In Rosen FS, Seligmann M, editor. Immunodeficiency Reviews. London, Harwood Academic Publications, 1990.

23. Hershfield MS, Chaffee S, Sorensen RU: Enzyme replacement therapy with polyethylene glycol-adenosine deaminase in adenosine deaminase deficiency: Overview and case reports of three patients, including two now receiving gene therapy. Pediatric Research 33(suppl 1):S42-S47; 1993

24. Fischer A, Hacein-Bey S, Cavazzana-Calvo M. Gene therapy of severe combined immunodeficiencies. Nature Reviews Immunology 2(8):615-21; 2002

25. Chaffee S, Mary A, Stiehm ER, et al. IgG antibody response to polyethylene glycol-modified adenosine deaminase in patients with adenosine deaminase deficiency. The Journal of clinical investigation 89(5):1643-51; 1992

26. Blaese RM, Culver KW, Chang L, et al. Treatment of severe combined immunodeficiency disease (SCID) due to adenosine deaminase deficiency with CD34+ selected autologous peripheral blood cells transduced with a human ADA gene. Amendment to clinical research project. Project 90-C-195, January 10, 1992. Human Gene Therapy 4(4):521-7; 1993

27. Joyner A, Keller G, Phillips RA, et al. Retrovirus transfer of a bacterial gene into mouse haematopoietic progenitor cells. Nature 305(5934):556-8; 1983

28. Bodine DM, McDonagh KT, Seidel NE, et al. Survival and retrovirus infection of murine hematopoietic stem cells in vitro: effects of 5-FU and method of infection. Experimental hematology 19(3):206-12; 1991

29. Wiginton DA, Adrian GS, Hutton JJ. Sequence of human adenosine deaminase cDNA including the coding region and a small intron. Nucleic acids research 12(5):2439-46; 1984

30. Wiginton DA, Kaplan DJ, States JC, et al. Complete sequence and structure of the gene for human adenosine deaminase. Biochemistry 25(25):8234-44; 1986

31. Parkman R, Gelfand EW, Rosen FS, et al. Severe combined immunodeficiency and adenosine deaminase deficiency. The New England journal of medicine 292(14):714-9; 1975

32. Tjonnfjord GE, Steen R, Veiby OP, et al. Evidence for engraftment of donor-type multipotent CD34+ cells in a patient with selective T-lymphocyte reconstitution after bone marrow transplantation for B-SCID. Blood 84(10):3584-9; 1994

33. Ferrari G, Rossini S, Giavazzi R, et al. An in vivo model of somatic cell gene therapy for human severe combined immunodeficiency. Science 251(4999):1363-66; 1991

34. Daddona PE, Mitchell BS, Meuwissen HJ, et al. Adenosine deaminase deficiency with normal immune function. An acidic enzyme mutation. The Journal of clinical investigation 72(2):483-92; 1983

35. Valentine WN, Paglia DE, Tartaglia AP, et al. Hereditary hemolytic anemia with increased red cell adenosine deaminase (45- to 70-fold) and decreased adenosine triphosphate. Science 195(4280):783-5; 1977

36. Kohn DB, Weinberg KI, Nolta JA, et al. Engraftment of genemodified cells from umbilical cord blood in Neonates with adenosine deaminase deficiency. Nature Medicine 1(10):101723; 1995

37. Hoogerbrugge PM, Beusechem VW, Fisher A, et al. Bone marrow gene transfer in three patients with adenosine deaminase deficiency. Gene Therapy 3:179-183; 1996

38. Lim B, Williams DA, Orkin SH. Retrovirus-mediated gene transfer of human adenosine deaminase: expression of functional enzyme in murine hematopoietic stem cells in vivo. Molecular and cellular biology 7(10):3459-65; 1987

39. Kiem HP, Andrews RG, Morris J, et al. Improved gene transfer into baboon marrow repopulation cells using recombinant human fibronectin fragment $\mathrm{CH}-296$ in combination with interleukin-6, stem cell factor, FLT-3 ligand, and megakaryocyte growth and development factor. Blood 92:1878-1886; 1998

40. Huhn RD, Tisdale JF, Agricola B, Metzger ME, Donahue RE, Dunbar CE. Retrovral marking and transplantation of rhesus hematopoietic cells by nonmyeloablative conditioning. Human Gene Therapy 10(11):1783-90; 1999

41. Fischer A, Hacein-Bey S, Le Deist F, et al. Gene therapy of severe combined immunodeficiencies. Immunological Reviews 178:13-20; 2000

Michael G. Woo is a second year Masters' student at the University of Manitoba, in the department of Pharmacology and Therapeutics. He holds a B.Sc. in Biology and Biochemistry from the University of Winnipeg. His research interests include the role of adenosine in the regulation of matrix metalloproteinases. 